\title{
Description of the Outcomes of Prior Authorization of Palivizumab for Prevention of Respiratory Syncytial Virus Infection in a Managed Care Organization
}

\author{
Brieana C. Buckley, PharmD; Derek Roylance, PharmD; Matthew P. Mitchell, PharmD, MBA; \\ Sushma M. Patel, PharmD; H. Eric Cannon, PharmD; and Jeffrey D. Dunn, PharmD, MBA
}

\begin{abstract}
BACKGROUND: Respiratory syncytial virus (RSV) is the leading cause of upper and lower respiratory tract infections in infants and young children. Most children are exposed to the virus before they are 2 years old and experience such symptoms as cough, fever, and irritability. In a select population of infants, the virus can cause hypoxemia and hospitalization. To avoid hospitalization, good infection control practices should be employed, and for those infants at high risk, prophylaxis with palivizumab is indicated. Palivizumab has been shown to reduce hospitalization rates in high-risk infants by $50 \%$. Because of the high cost of palivizumab, it is prudent to use this medication in the population in which it will be most effective. The American Academy of Pediatrics (AAP) established the criteria for those infants who would benefit the most from palivizumab prophylaxis, and these criteria were the foundation for a prior authorization (PA) program to determine coverage of palivizumab in a health plan of approximately 500,000 members.
\end{abstract}

OBJECTIVE: To (a) analyze the appropriateness of this PA program for palivizumab used prophylactically for RSV, and (b) determine the financial cost associated with the medication and disease for this health plan.

METHODS: A 3-year, retrospective study was conducted from the 20052006 RSV season through the 2007-2008 season. The primary endpoint outcome was the hospitalization rate associated with RSV infection. Secondary endpoints included the cost of palivizumab and RSV-related emergency room (ER) utilization. Infants were placed into 2 groups: those who received PA approval for use of palivizumab and those who were denied coverage in the PA process. Disease-related hospitalization and ER visits were identified by at least 1 administrative claim containing either a primary or secondary ICD-9-CM code for any of the following: RSV (079.6), acute bronchiolitis caused by RSV (466.11), or pneumonia caused by RSV (480.1). Drug cost was defined as the health plan's allowed amount, which is based on a predefined fee schedule for the Current Procedural Terminology (CPT) code 90378 for palivizumab. Hospital and ER costs are the health plan allowed amounts (health plan plus member cost) based on the reimbursement rates determined by diagnosis related group (DRG) and other coding, and the plan-allowed amount based on DRGs includes all services and drugs provided in the specific encounter. Drug cost avoided was calculated as the average cost of palivizumab treatment per episode multiplied by the number of infants denied coverage of palivizumab over the 3-year study period.

RESULTS: Over 3 RSV seasons through May 2008, the PA program received 1,090 requests for coverage of palivizumab, of which $348(31.9 \%)$ were denied. Of 742 PA-approved infants, 629 received at least 1 dose of palivi-

Note: This article is the subject of an editorial that appears on pages 59-66 of this issue. zumab. The mean (SD) gestational age of the PA-denied group was 34.4 (2.5) weeks versus 32.5 (4.0) weeks for the PA-approved group $(P<0.001)$. In the PA-denied group, 14 infants (4.0\%) were subsequently hospitalized with an RSV infection, and $5(1.4 \%)$ had an RSV-related ER visit versus $40(6.4 \%)$ hospitalized and $14(2.2 \%)$ with ER visits for infants in the PA-approved group ( $P=0.055$ and $P=0.019$, respectively); $15(4.3 \%)$ of the PA-denied group had either a hospitalization or an ER visit versus 42 $(6.6 \%)$ in the PA-approved group $(P=0.060)$. One patient in the palivizumab PA-approved group died. Over the 3 RSV seasons, the mean number of palivizumab doses and mean allowed palivizumab cost per treatment episode (per infant per season) were 3.64 and $\$ 6,950$, respectively, and the average allowed palivizumab cost was $\$ 7,702$ per utilizing infant. Total per infant costs for palivizumab, RSV hospitalizations, and RSV-related ER visits were $\$ 8,534$ for infants receiving palivizumab compared with $\$ 223$ for those denied palivizumab coverage $(P=0.002)$. Drug cost avoidance associated with the PA program was estimated to be $\$ 2,418,600$ (348 infants times $\$ 6,950$ palivizumab cost per episode) over the 3 RSV seasons.

CONCLUSION: In a 500,000-member health plan, a PA program to restrict palivizumab use in accordance with AAP recommendations was associated with estimated palivizumab drug cost avoidance of more than \$2.4 million over 3 years. There was no significant difference in the RSV-related hospitalization rate for the PA-denied versus the PA-approved groups, but the PA-denied group had a slightly lower rate of RSV-related ER visits.

J Manag Care Pharm. 2010;16(1):15-22

Copyright $\odot$ 2010, Academy of Managed Care Pharmacy. All rights reserved.

\section{What is already known about this subject}

- Respiratory syncytial virus (RSV) is the most common cause of bronchiolitis and pneumonia among infants and children younger than 1 year of age.

- Palivizumab has been shown in studies to reduce the frequency of RSV-related hospitalization in high-risk infants by $50 \%$.

- Palivizumab is an expensive medication, with wholesale acquisition cost (WAC) in 2009 of $\$ 955$ per $50 \mathrm{mg}(0.5 \mathrm{~mL})$ vial and $\$ 1,802$ per $100 \mathrm{mg}(1 \mathrm{~mL})$ vial.

- Joffe et al. (1999) found that the cost per avoided hospitalization associated with immunoprophylaxis with palivizumab ranged from $\$ 12,000$ in the highest-risk infants to $\$ 420,000$ in lowerrisk infants-gestational age 33-36 weeks, used less than 28 days oxygen in the neonatal intensive care unit (NICU), and were discharged between December and August (1995 dollars). 


\section{Description of the Outcomes of Prior Authorization of Palivizumab for Prevention of Respiratory Syncytial Virus Infection in a Managed Care Organization}

\section{What this study adds}

- This is the first published study to determine rates of hospitalizations, emergency room (ER) visits, and drug cost avoidance associated with the application of prior authorization (PA) criteria for coverage determination of palivizumab.

- Over 3 RSV seasons ending in May 2008 in a 500,000-member health plan, drug cost avoidance associated with the PA program was estimated to be $\$ 2,418,600$ for 348 infants denied coverage of palivizumab, with no significant difference in the rate of RSVrelated hospitalization or ER visits between the PA-denied group $(4.3 \%, n=15)$ and the PA-approved group $(6.6 \%, n=42)$.

- Drug cost avoidance can be realized through application of PA criteria to determine appropriate use of palivizumab, and there was no significant difference in the proportion of infants with at least 1 RSV hospitalization, comparing infants who received palivizumab (6.4\%) with infants who were denied palivizumab $(4.0 \%, P=0.055)$

$\mathrm{R}$ espiratory syncytial virus (RSV) is the most common cause of bronchiolitis and pneumonia among infants and children under 1 year of age. ${ }^{1}$ Illness begins most frequently with fever, runny nose, cough, and wheezing. During their first RSV infection, between $25 \%$ and $40 \%$ of infants and young children have signs or symptoms of bronchiolitis or pneumonia, and $0.5 \%$ to $2 \%$ require hospitalization. ${ }^{2}$ Most children recover from illness in 8 to 15 days. ${ }^{2}$ The majority of children hospitalized for RSV infection are younger than 6 months of age. ${ }^{2}$ RSV also causes infections throughout life, usually associated with moderate-tosevere cold-like symptoms; however, severe lower respiratory tract disease may occur at any age, especially among the elderly or among those with compromised cardiac, pulmonary, or immune systems. ${ }^{3}$ In temperate climates, RSV infections usually occur during annual community outbreaks, often lasting 4 to 6 months, during the late fall, winter, or early spring months. The timing and severity of outbreaks in a community vary from year to year. ${ }^{4,5}$ The typical RSV season in Utah, where the present study was conducted, generally begins in December and lasts through April. SelectHealth, a 500,000-member integrated health system based in Salt Lake City, defines the RSV season based on local epidemiological data.

Development of an RSV vaccine is a high research priority, but one is not yet available. Current prevention options include good infection-control practices and palivizumab (Synagis), a monoclonal antibody. Palivizumab works by exhibiting neutralizing and fusion-inhibitory activity against RSV. This mechanism has been shown to inhibit RSV replication in laboratory experiments. ${ }^{6}$ Palivizumab can be given during the RSV outbreak season to prevent serious lower respiratory tract disease caused by RSV in pediatric patients at high risk for serious RSV disease (e.g., those with chronic lung disease [CLD], infants born prematurely with or without CLD, and those with congenital heart disease [CHD]). ${ }^{6}$ Palivizumab has no potential for infectious contamination, is given intramuscularly, and does not interfere with the timing of administration of vaccines. ${ }^{7}$ The first dose should be administered prior to commencement of the RSV season, and high-risk infants born during the RSV season may receive palivizumab prophylaxis from birth. At the time that the present study was conducted, high-risk infants received palivizumab until the end of the RSV season and thus could have received 1 to 5 injections depending on their month of birth.

The cost of RSV prophylaxis versus the cost of hospitalization continues to be debated. According to the National Institutes of Health, each year up to 125,000 infants under 1 year of age are hospitalized because of severe RSV disease. ${ }^{8}$ Palivizumab is a costly drug with a wholesale acquisition cost (WAC) in 2009 of $\$ 955$ per $50 \mathrm{mg}(0.5 \mathrm{~mL})$ vial or $\$ 1,802$ per $100 \mathrm{mg}(1.0 \mathrm{~mL})$ vial, ${ }^{9}$ leading to debate regarding which children should receive such prophylaxis. Lofland et al. (2000) found the incremental cost per RSV infection episode avoided ranged from $\$ 2,702$ to $\$ 79,706$ when the cost per course of palivizumab was $\$ 4,500$. When the cost per course of palivizumab was $\$ 2,500$, cost per RSV infection avoided ranged from $\$ 0$ to $\$ 39,591 . .^{10}$ An analysis by Joffe et al. (1999) that explored the cost-effectiveness of palivizumab when targeting high-risk patient subgroups found that the cost per avoided RSV hospitalization was estimated at approximately $\$ 12,000$ in 1995 dollars in the highest-risk patient subgroup (gestational age 23-32 weeks who received at least 28 days of oxygen in the neonatal intensive care unit [NICU] and were discharged from the NICU between September and November). ${ }^{11}$ However, the cost per avoided hospitalization was as high as $\$ 420,000$ in the subgroup of infants whose gestational age was 33-36 weeks, who received less than 28 days oxygen in the NICU, and who were discharged between December and August, demonstrating the high cost of prophylaxis in lower-risk subgroups. ${ }^{11}$

Due to the high cost of palivizumab and its special target population, palivizumab is a good candidate for prior authorization (PA). According to a survey of health plans conducted in December $2008,81 \%$ of respondents indicated that they currently require PA for palivizumab..$^{12}$ In order to ensure that palivizumab is used appropriately, SelectHealth has required PA for palivizumab since the time that it became commercially available. The SelectHealth PA criteria that were in place at the time of the present study were based on a high-risk population subset (Figure 1) that corresponds to the American Academy of Pediatrics (AAP) 2006 guidelines (Appendix). ${ }^{13}$ The objective of the present study was to analyze in a real-world setting the outcomes of application of PA criteria required by SelectHealth for palivizumab when given prophylactically for RSV, including the financial cost associated with the disease. Guidelines by the AAP define a select population for which palivizumab is recommended. Many health plans have adopted the AAP guidelines to create coverage policies for this select patient population. To our knowledge, this is the first 


\section{Description of the Outcomes of Prior Authorization of Palivizumab for Prevention of Respiratory Syncytial Virus Infection in a Managed Care Organization}

\section{FIGURE 1 SelectHealth Prior Authorization Criteria for Use of Palivizumaba}

Prior authorization approval and coverage of palivizumab are contingent on the member meeting 1 of the 3 following criteria:

1. Less than 2 years of age with CLD requiring medical therapy (such as supplemental oxygen, bronchodilator, diuretic or corticosteroid therapy) within 6 months before start of RSV season, or the infant has hemodynamically significant CHD

2. Infants born less than or equal to 32 weeks of gestation with or without CLD

a. Major risk factors: gestational age and chronologic age at start of season

b. Born less than 28 weeks gestational age and less than 12 months of age

c. Born 29-32 weeks gestational age and less than 6 months of age before start of RSV season

3. Infants born between 32-35 weeks gestational age and less than 6 months old at start of RSV season with 2 or more risk factors:

- Day care attendance

- School-age siblings

- Exposure to environmental pollutants (including secondhand smoke)

aThese criteria have been in effect at SelectHealth since 2002 and are based on the recommendations of the AAP13 for the use of palivizumab (see Appendix). The SelectHealth preauthorization form and criteria were revised in September 200923 to incorporate the new guidelines published online by the AAP in September 2009.22 $A A P=$ American Academy of Pediatrics; $C H D=$ congenital heart disease; $C L D=$ chronic lung disease; $R S V=$ respiratory syncytial virus.

published analysis of the outcomes associated with a health plan's PA criteria for coverage determination of palivizumab. The results of the present analysis were anticipated to provide assistance in refining the PA criteria for the appropriate use of palivizumab in this 500,000-member health plan.

\section{Methods}

This exploratory and descriptive study was a retrospective analysis of an administrative claims database. All members enrolled in the health plan during any of 3 RSV seasons-2005-2006, 20062007, or 2007-2008-were eligible for the study. Continuous enrollment was not part of the inclusion criteria because eligible infants could be born during the study period, limiting the number of months they were enrolled. Infants were included in the analysis if they had a PA request for palivizumab submitted for 1 or more of the 3 RSV seasons. PA requests are submitted to the health plan via fax by health care physicians or specialty pharmacies. These requests could be submitted prior to or during the RSV season. If approved, authorizations were entered for infants to receive up to 5 doses of palivizumab from November through April of the requested RSV season. All PA requests were reviewed by a clinical pharmacist, and benefit determination was based on the health plan's criteria (Figure 1).

The PA-approved group was composed of infants who met the PA criteria, were approved for palivizumab coverage, and received at least 1 dose of palivizumab (Current Procedural Terminology [CPT] code 90378 [RSV immune globulin, intramuscular use]; national drug code [NDC] and Healthcare Common Procedure Coding System [HCPCS] codes are not used for reimbursement of palivizumab in this health plan). For those infants who received palivizumab, the drug was covered under the medical benefit and dispensed through a specialty pharmacy or billed through a home health provider or a physician's office. Medical claims are reimbursed based on the submitted Health Insurance Claim Form (CMS-1500), which requires the use of either a HCPCS or CPT code, and there is no field designated for NDC numbers on the CMS-1500. Based on these limitations, SelectHealth collects NDCs when possible but chooses to reimburse based on the CPT code. The PA-denied group in this study was composed of those infants who did not meet the PA requirements and whose requests for coverage of palivizumab were denied.

Disease-related utilization was defined as any claim billed from an inpatient hospital with either a primary or secondary International Classification of Diseases, Ninth Revision, Clinical Modification (ICD-9-CM) code for any of the following: RSV (079.6), acute bronchiolitis caused by RSV (466.11), or pneumonia caused by RSV (480.1). Inpatient visits were identified by CPT codes 99221-99239 (indicating inpatient evaluation and management). Emergency room (ER) visits were identified as services billed from an ER with corresponding CPT codes 99281-99285 (indicating ER evaluation and management). Gender, gestational age, and palivizumab utilization data were obtained from the health plan's electronic claims database.

Outcome measures included incidence and cost of inpatient hospital stays and ER visits, the number of palivizumab doses per infant, the cost of palivizumab, and deaths. ER visits and inpatient hospitalizations were included in the analysis if they occurred during the same season that palivizumab was requested. Hospital and ER costs were expressed as total allowed amount, defined as the contracted reimbursement rate based on submitted diagnosis related group (DRG) codes per associated RSV treatment episode. Payment based on DRG includes all services and drugs provided to the infants related to the specific inpatient or ER encounter. Drug cost avoided was calculated as the average cost of palivizumab per treatment episode multiplied by the number of infants denied coverage of palivizumab over the 3-year study 


\section{Description of the Outcomes of Prior Authorization of Palivizumab for Prevention of Respiratory Syncytial Virus Infection in a Managed Care Organization}

\section{TABLE 1 Drug, Hospital, and ER Utilization and Costs for PA-Approved}

Versus PA-Denied Infants for 3 RSV Seasons (2005-2008)

\begin{tabular}{|c|c|c|c|}
\hline & Palivizumab Approved & Palivizumab Not Approved & $P$ Value $^{\mathrm{a}}$ \\
\hline \multicolumn{4}{|l|}{ Entire sample } \\
\hline Number of coverage requests & 742 & 348 & \\
\hline Number of infants & 629 & 348 & \\
\hline Number (\%) female & $252(40.1 \%)$ & $160(46.0 \%)$ & 0.422 \\
\hline Mean [SD] gestational age & $32.5[4.0]$ & $34.4[2.5]$ & $<0.001$ \\
\hline Direct drug cost of palivizumab & $\$ 4,844,435$ & $\$ 0$ & \\
\hline \multicolumn{4}{|l|}{ Patients with at least 1 RSV hospitalization ${ }^{b}$} \\
\hline Number (\%) of infants & $40(6.4 \%)$ & $14(4.0 \%)$ & 0.055 \\
\hline Number (\%) female & $12(30 \%)$ & $5(35.7 \%)$ & 0.314 \\
\hline Mean [SD] gestational age & $33.5[4.6]$ & $34.9[3.1]$ & 0.227 \\
\hline RSV-related inpatient hospital costs & $\$ 517,418$ & $\$ 73,159$ & \\
\hline Cost per hospitalized infant & $\$ 12,935$ & $\$ 5,225$ & \\
\hline \multicolumn{4}{|l|}{ Patients with at least 1 RSV-related ER visit ${ }^{\text {b }}$} \\
\hline Number (\%) of infants & $14(2.2 \%)$ & $5(1.4 \%)$ & 0.019 \\
\hline Number (\%) female & $5(35.7 \%)$ & $1(20 \%)$ & 0.315 \\
\hline Mean [SD] gestational age & $34.0[3.8]$ & $36.5[2.0]$ & 0.131 \\
\hline RSV-related ER costs & $\$ 5,918$ & $\$ 4,292$ & \\
\hline Mean cost per infant with ER visit & $\$ 423$ & $\$ 858$ & \\
\hline \multicolumn{4}{|l|}{ Whole-sample costs } \\
\hline Total costs & $\$ 5,367,771$ & $\$ 77,451$ & \\
\hline Distinct infants with hospitalization or ER visit & $42(6.6 \%)$ & $15(4.3 \%)$ & 0.060 \\
\hline \multicolumn{4}{|l|}{ Per infant costs } \\
\hline Palivizumab & $\$ 7,701.80$ & $\$ 0$ & \\
\hline Inpatient hospital & $\$ 822.60$ & $\$ 210.23$ & 0.015 \\
\hline ER & $\$ 9.41$ & $\$ 12.33$ & 0.141 \\
\hline Total costs & $\$ 8,533.82$ & $\$ 222.56$ & 0.002 \\
\hline
\end{tabular}

period. Deaths were verified with state vital statistics records that are exchanged electronically once per month between the state of Utah and the health plan. State-reported deaths were queried against infants included in both study groups. Mean gestational age for each infant subgroup was calculated. Between-group differences in gender, gestational age, inpatient hospitalization, and ER visit rates were assessed for statistical significance using Fisher's Exact test for proportions and the Student's t-test for means. The significance level was set at 0.05 . Analyses were performed by an internal data analyst using Analysis ToolPak 2003. This study was approved by the Intermountain Healthcare Office of Research and Institutional Review Board.

\section{Results}

Providers submitted PA requests for palivizumab for a total of 1,090 infants over the 3 RSV seasons, of which 742 (68.1\%) were approved and 348 (31.9\%) were denied. Of the 742 PA approvals, 629 infants had at least 1 paid claim for palivizumab in their electronic claims record and were included in the analysis (Table
1); the 113 infants without a paid claim for palivizumab were excluded from the analysis. The cohort of PA-approved infants was $40.1 \%$ female $(n=252)$ compared with $46.0 \%(n=160)$ in the PA-denied cohort $(P=0.422)$. The mean (SD) gestational age was lower in the PA-approved group (32.5 [4.0] weeks) compared with the PA-denied group (34.4 [2.5] weeks, $P<0.001$ ).

Of the 348 infants who were denied coverage for palivizumab, 5 (1.4\%) had a documented RSV-related ER visit, and 14 (4.0\%) had an RSV-related hospital stay (Table 1). Four of the 5 infants with an ER visit had subsequent inpatient hospital claims for RSV. Of the 629 infants who met the PA criteria and received palivizumab, 14 (2.2\%) had an ER visit, and 40 (6.4\%) were hospitalized for an RSV infection. Of the 14 infants with an ER visit, 12 also had resulting inpatient hospitalizations. One infant who received palivizumab died of respiratory failure. There were no deaths in the PA-denied group. The mean gestational ages were not significantly different for the PA-denied group compared with the PA-approved group for the infants who had at least 1 RSVrelated hospitalization or at least 1 RSV-related ER visit (Table 1). 
Description of the Outcomes of Prior Authorization of Palivizumab for Prevention

of Respiratory Syncytial Virus Infection in a Managed Care Organization

\section{TABLE 2 Utilization and Cost of Palivizumab by RSV Season}

\begin{tabular}{|c|c|c|c|c|}
\hline RSV Season & $2005-2006$ & 2006-2007 & $2007-2008$ & Total \\
\hline Number of infants who received palivizumab & 239 & 229 & 229 & $\begin{array}{l}629 \text { infants } \\
697 \text { episodes }^{\mathrm{a}}\end{array}$ \\
\hline Number of doses & 859 & 855 & 826 & 2,540 \\
\hline Average doses per episode (RSV season) & 3.59 & 3.73 & 3.60 & $\begin{array}{c}4.04 \text { per infant } \\
3.64 \text { per episode }\end{array}$ \\
\hline Total palivizumab cost & $\$ 1,500,040$ & $\$ 1,740,939$ & $\$ 1,603,456$ & $\$ 4,844,435$ \\
\hline Palivizumab cost per infant & $\$ 6,276$ & $\$ 7,602$ & $\$ 7,002$ & $\begin{array}{c}\$ 7,702 \text { per infant } \\
\$ 6,950 \text { per episode }\end{array}$ \\
\hline
\end{tabular}

aThe unique patient (infant) count is not equal to the total count of episodes because some infants received palivizumab in more than 1 RSV season.

$R S V=$ respiratory syncytial virus.

Between-group comparisons showed that the proportion of infants with an ER visit was significantly lower in the PA-denied group $(1.4 \%, \mathrm{n}=5)$ than in the PA-approved group $(2.2 \%, \mathrm{n}=14$; $P=0.019)$. Additionally, of those who were denied palivizumab, per-infant inpatient hospital costs $(\$ 210.23)$ and total costs (\$222.56) were significantly lower for the PA-denied group than for the PA-approved group ( $\$ 822.60$ and $\$ 8,533.82 ; P=0.015$ and $P=0.002$, respectively). The mean gestational age of the PA-approved group (32.5 weeks) was significantly lower than the mean gestational age of the PA-denied group (34.4 weeks, $P<0.001$ ).

The total cost of palivizumab over the 3 seasons was $\$ 4,844,435$ ( $\$ 7,702$ per unique infant or $\$ 6,950$ per treatment episode; Table 2). Among those who received palivizumab, perinfant costs for the drug increased from the 2005-2006 to the 2007-2008 season. Drug cost avoidance associated with the PA program over the 3 RSV seasons was estimated to be $\$ 2,418,600$ (348 PA denials multiplied by $\$ 6,950$ per palivizumab episode).

\section{Discussion}

This study assessed the experience of a 500,000-member health plan in implementing guideline-based coverage criteria for the use of palivizumab. Of the infants who were denied palivizumab coverage, $4.3 \%$ were hospitalized or had an ER visit, compared with $6.6 \%$ for the infants who were approved for coverage of palivizumab $(P=0.060)$. The rates of RSV-related hospitalization for PA-approved infants and PA-denied infants did not significantly differ (6.4\% vs. $4.0 \%, P=0.055)$. Our RSV-related hospitalization rate of $6.4 \%$ for PA-approved infants and $4.0 \%$ for PA-denied infants compares with an average annual RSV-related hospitalization rate of $1.7 \%$ for children less than 6 months of age in the sample studied by Hall et al. (2009) that included both high-risk and low-risk children younger than 5 years of age. ${ }^{14}$ Hall et al. found that the RSV-related hospitalization rate was $1.7 \%$ for the youngest infants ( $0-5$ months of age) but dropped off dramatically to $0.5 \%$ for 6 to 11 months of age, $0.3 \%$ for 12 to 23 months of age, and $0.04 \%$ for 24 to 59 months of age. Overall for children aged 0 to 59 months, $0.3 \%$ were hospitalized and $2.8 \%$ were seen in ERs for the treatment of RSV.
For patients receiving palivizumab, only 2 randomized controlled trials have investigated the efficacy of palivizumab in reducing RSV-associated hospitalizations. The IMpact-RSV clinical trial results for the 1996-1997 RSV season for children with either prematurity up to 35 weeks gestation or bronchopulmonary dysplasia/chronic lung disease (BPD/CLD) showed that the palivizumab-treated group had a $4.8 \%$ rate of RSV-related hospitalization compared with a $10.6 \%$ rate for the placebo group $(P<0.001) .15$

Broad variability has been demonstrated in observational analyses reporting hospitalization rates associated with RSV. Estimates of RSV-related hospitalizations by the Institute of Medicine (IOM; 1985) suggested that only 55,000 U.S. infants younger than 1 year of age were hospitalized for bronchiolitis or pneumonia that might be RSV-related. ${ }^{16}$ Shay et al. (1999) later reported in an analysis of U.S. National Hospital Discharge Survey (NHDS) data for 1980-1996 that the rate of hospitalization for bronchiolitis for children less than 1 year of age had increased more than 2-fold over the 17-year study period, from 12.9 per 1,000 in 1980 to 31.2 per 1,000 in 1996; $57 \%$ of bronchiolitisassociated hospitalizations occurred among infants younger than 6 months of age and 81\% among those younger than 1 year of age..$^{17}$ More recently, NHDS data for 1997-2002 show that RSV-related hospitalization rates increased an additional 25\% among infants less than 1 year of age. ${ }^{18}$ Shay et al. theorized that the burden of RSV may be increasing due to an increase in the prevalence of child care for younger children and revised criteria for hospitalization; diagnostic coding and the virulence of RSV strains were probably not factors. However, only attendance in child care was found to be a risk factor in the reported incidence of RSV-related hospitalization.

In our study, the observed RSV hospitalization rates are higher than reported in the IMpact-RSV clinical trials. One possible explanation is the demonstrated increasing rate of reported RSVrelated hospitalizations since the 1980s. While national rates of RSV-related hospitalization range from $0.5 \%$ to $2 \%$, there is significant geographical variation. Demographically, Utah residents have a higher proportion of children under the age of $5(9.8 \%)$ and a greater number of persons per household (3.13) compared 


\section{Description of the Outcomes of Prior Authorization of Palivizumab for Prevention of Respiratory Syncytial Virus Infection in a Managed Care Organization}

with national data (6.9\% and 2.59, respectively). ${ }^{19}$ More young children in our population could contribute to higher proportion of infants with school-aged siblings, more prevalent day care attendance, or crowded living conditions. Each of these have been identified as risk factors for severe RSV disease. ${ }^{20,21}$

Our observational analysis demonstrated significantly lower costs of hospitalization for the PA-denied group compared with the PA-approved group. Common reasons for denial of PA submissions within SelectHealth include infants older than 2 years of age, no documented CHD or CLD requiring continued medical therapy, gestational age greater than 35 weeks, or gestational age 32-35 weeks without the presence of risk factors (Figure 1). Each of these characteristics imposes a greater risk for severe RSV infection. Based on the reasons for PA denial, the PA-denied group is a more healthy population. This is supported by the comparison of the gestational age, where the PA-approved group had a significantly lower gestational age than the PA-denied group. However, we found no difference in ER costs for the PA-denied group compared with the PA-approved group. We did not measure the severity of illness in the infants who had ER visits or hospitalization.

Our outcomes analysis found value in this PA program for palivizumab based on the 2006 AAP criteria. The AAP released modified recommendations for the use of palivizumab in September 2009.22 The new recommendations were updated in an effort to ensure optimal balance of benefit and cost from this expensive intervention based on additional data on risk factors for identifying children who are at increased risk of serious RSV lower respiratory tract disease. The 2009 AAP recommendations emphasize 4 main points.

1. Initiation and termination of prophylaxis have been modified to reflect RSV seasonality in different geographic locations; emphasis has been placed on the use of local epidemiologic data to define the peak outbreak months. Early initiation or continuation of therapy during months in which RSV is not widely circulating provides little benefit.

2. The dosing schedule remains unchanged for infants before 32 weeks 0 days gestation who have CHD or CLD. The recommendation for a maximum number of 5 doses is emphasized for all geographic locations for infants meeting these criteria.

3. No more than 3 doses are recommended for infants with a gestational age between 32 weeks 0 days and 34 weeks 6 days who are without hemodynamically significant CHD or CLD who otherwise qualify for prophylaxis.

4. Infants born between 32 weeks 0 days and 34 weeks 6 days gestation qualify for prophylaxis if they have at least 1 of 2 risk factors but should receive prophylaxis only until they reach 90 days of age or a maximum of 3 doses (whichever occurs first). Risk factors have been modified to include only the following: the infant is in child care or 1 or more siblings or other children younger than age 5 live permanently in the child's household. 22
SelectHealth examined the revised AAP recommendations released online in September 2009 and incorporated the new criteria in the PA process for the 2009-2010 RSV season. ${ }^{23}$ The only modification that directly impacts the PA criteria is the recommendation for infants born between 32 weeks 0 days and 34 weeks 6 days gestation. Infants requesting coverage of palivizumab based on this criteria will be approved only if they have 1 of the following risk factors: either the infant is in child care or has siblings younger than age 5 in their household. If palivizumab is approved, coverage is limited to no more than 3 doses or until 90 days of age, whichever occurs first. The SelectHealth PA form consistent with the revised AAP recommendations was updated in September 2009 and is available via the SelectHealth Web portal at www.selecthealth.org/pharmacy. ${ }^{23}$

\section{Limitations}

First, relevant infant-specific data, such as history of underlying conditions that predispose an infant to respiratory complications (e.g., neurologic disease in very low birth weight infants, number of young siblings, child care center attendance, and exposure to tobacco smoke in the home), cannot be identified through electronic claims data and may affect the findings of this study. Second, this study is an exploratory descriptive analysis and its observational design prevents attribution of causality. Third, we did not assess compliance with the recommended number of injections or the vaccination schedule and did not require continuous eligibility for the infants; however, for SelectHealth the annual renewal rate has been $95.5 \%$. Thus, we do not know if infants in the sample were enrolled in the health plan for the entire RSV season when palivizumab was requested. Fourth, the present study assumed that a diagnosis of RSV recorded in an administrative claim is accurate. Fifth, this study was an observational analysis that examined only the cost of RSV-related hospitalization and RSV-related ER visits, not total RSV-related costs including office visits and outpatient treatment costs.

\section{Conclusion}

RSV infections are common among young infants and can result in hospitalization for those at high risk. Palivizumab is an expensive medication that when given as prophylaxis can reduce hospitalization rates. Because of the high direct drug cost of palivizumab, PA criteria have been established to restrict coverage of the medication to the infants who would benefit most. Our 3-year retrospective analysis showed that the PA program was associated with significant palivizumab drug cost avoidance of more than $\$ 2.4$ million in a 500,000-member health plan. These drug cost savings were achieved without apparent adverse clinical outcomes in the rate of RSV-related hospitalization or RSVrelated ER visits. Although exploratory, these results suggest that implementation of PA criteria for palivizumab in accordance with clinical guidelines is associated with significant drug cost avoidance without an increase in the cost or the incidence of ER visits or inpatient hospitalizations associated with RSV infections. 


\section{Description of the Outcomes of Prior Authorization of Palivizumab for Prevention of Respiratory Syncytial Virus Infection in a Managed Care Organization}

\section{Authors}

BRIEANA C. BUCKLEY, PharmD, is Clinical Pharmacy Coordinator; DEREK ROYLANCE, PharmD, was Pharmacy Resident at the time of this study; MATTHEW P. MITCHELL, PharmD, MBA, is Manager of Pharmacy Services; SUSHMA M. PATEL, PharmD, is Clinical Pharmacy Coordinator; H. ERIC CANNON, PharmD, is Chief of Pharmacy; and JEFFREY D. DUNN, PharmD, MBA, is Formulary and Contract Manager, SelectHealth, Salt Lake City, Utah.

AUTHOR CORRESPONDENCE: Brieana C. Buckley, PharmD, SelectHealth, 4646 W. Lake Park Blvd., Salt Lake City, UT 84120 Tel.: 801.442.7797; E-mail: Brieana.cox-buckley@selecthealth.org.

\section{DISCLOSURES}

This study had no external funding. Cannon reported participation in a continuing education program on RSV that was funded through an unrestricted education grant from MedImmune. The other authors disclosed no financial or nonfinancial conflicts of interests related to any company, product, or service discussed in this article.

Buckley and Cannon were primarily responsible for the study concept and design. Roylance and Buckley were primarily responsible for data collection and interpretation with assistance from Patel and Mitchell. Roylance and Dunn were primarily responsible for writing the first version of the manuscript, and Buckley and Mitchell revised the manuscript.

\section{ACKNOWLEDGEMENTS}

Matt Speckman, Senior Outcomes Analyst, assisted with the data analyses for this research.

\section{REFERENCES}

1. Black CP. Systematic review of the biology and medical management of respiratory syncytial virus infection. Respir Care. 2003;48(3):209-31.

2. Centers for Disease Control and Prevention. Respiratory syncytial virus infection (RSV). October 23, 2008. Available at: http://www.cdc.gov/rsv/. Accessed November 29, 2009.

3. Falsey AR, Hennessey PA, Formica MA, Cox C, Walsh EE. Respiratory syncytial virus in the elderly and high-risk adults. $N$ Engl J Med. 2005;352(17):1749-59. Available at: http://content.nejm.org/cgi/ reprint/352/17/1749.pdf. Accessed November 29, 2009.

4. Hall CB. Respiratory syncytial virus and parainfluenza virus. N Engl J Med. 2001;344(25):1917-28. Available at: http://content.nejm.org/cgi/ reprint/344/25/1917.pdf. Accessed November 29, 2009.

5. Krilov LR. Palivizumab in the prevention of respiratory syncytial virus disease. Expert Opin Biol Ther. 2002;2:763-69.

6. Product label. Synagis (palivizumab) for intramuscular injection. March 2009. Available at: http:/www.medimmune.com/pdf/products/synagis_ pi.pdf. Accessed November 29, 2009

7. ThomsonHealthcare. Micromedex® Healthcare Series (electronic version), Available at: http://www.thomsonhc.com. Accessed November 29, 2009.
8. National Institute of Allergy and Infections Disease. Respiratory syncytial virus quick facts. December 2, 2008. Available at: http://www3.niaid.nih. gov/topics/rsv/understanding/quickFacts.htm. Accessed November 29, 2009. 9. Drug Codes.com. Drug coding and pricing guide. Available at: www. drugcodes.com. Accessed November 29, 2009.

10. Lofland JH, O'Connor JP, Chatterton ML, et al. Palivizumab for respiratory syncytial virus prophylaxis in high-risk infants: a cost-effectiveness analysis. Clin Ther. 2000;22(11):1357-69.

11. Joffe S, Ray GT, Escobar GJ, Black SB, Lieu TA. Cost-effectiveness of respiratory syncytial virus prophylaxis among preterm infants. Pediatrics. 1999;104(3 Pt 1):419-27.

12. EMD Serono. Serono Injectables Digest, 5th ed. April 2009. Available at: https://olr.dwainc.com/2559Digest/PDF/EMDSerono5th EditionInjectablesDigest.pdf. Accessed November 29, 2009.

13. Subcommittee on Diagnosis and Management of Bronchiolitis. Clinical practice guideline. Diagnosis and management of bronchiolitis. Pediatrics. 2006;118(4):1774-93. Available at: http://pediatrics.aappublications.org/cgi/ reprint/118/4/1774. Accessed November 29, 2009.

14. Hall CB, Weinberg GA, Iwane MK, et al. The burden of respiratory syncytial virus infection in young children. N Engl J Med. 2009;360(6):588-98. Available at: http://content.nejm.org/cgi/reprint/360/6/588.pdf. Accessed November 29, 2009.

15. IMpact-RSV Study Group. Palivizumab, a humanized respiratory syncytial virus monoclonal antibody, reduces hospitalization from respiratory syncytial virus infection in high-risk infants. Pediatrics. 1998;102(3 Pt 1): 531-37.

16. Institute of Medicine. Prospects for immunizing against respiratory syncytial virus. In: New Vaccine Development: Establishing Priorities. Vol 1. Washington: National Academies Press; 1985:397-409.

17. Shay DK, Holman RC, Newman RD, et al. Bronchiolitis-associated hospitalizations among US children, 1980-1996. JAMA. 1999;282(15):1440-46. Available at: http://jama.ama-assn.org/cgi/reprint/282/15/1440. Accessed November 24, 2009.

18. McLaurin KK, Leader S. Growing impact of RSV hospitalizations among infants in the US, 1997-2002 (abstract 936). Poster presented at: Pediatric Academic Societies Annual Meeting, May 14-17, 2005; Washington D.C.

19. U.S. Census Bureau. State and County Quick Facts. Data derived from Population Estimates, Census of Population and Housing, Small Area Income and Poverty Estimates, State and County Housing Unit Estimates, County Business Patterns, Nonemployer Statistics, Economic Census, Survey of Business Owners, Building Permits, Consolidated Federal Funds Report. Available at: http://quickfacts.census.gov/qfd/states/49000.html. November 29, 2009.

20. Figueras-Aloy J, Carbonell-Estrany X, Quero J, for the IRIS Study Group. Case-controlled study of the risk factors linked to respiratory syncytial virus infection requiring hospitalization in premature infants born at a gestational age of 33-35 weeks in Spain. Pediatr Infect Dis J. 2004;23(9):815-20.

21. Law BJ, Langley JM, Allen U, et al. The Pediatric Investigators Collaborative Network on Infections in Canada study of predictors of hospitalization for respiratory syncytial virus infection for infants born at 33 through 35 weeks of gestation. Pediatr Infect Dis J. 2004;23(9):806-14.

22. Committee on Infectious Diseases. Policy statement-modified recommendations for use of palivizumab for prevention of respiratory syncytial virus infections. Pediatrics. 2009:124(6): 1-8. Available at: http://pediatrics. aappublications.org/cgi/reprint/peds.2009-2345vl. Accessed November 29, 2009.

23. SelectHealth. Preauthorization form: Synagis (palivizumab). September 17, 2009. Available at: http://selecthealth.org/Static/Files/synagis.pdf. Accessed November 29, 2009. 
Description of the Outcomes of Prior Authorization of Palivizumab for Prevention

of Respiratory Syncytial Virus Infection in a Managed Care Organization

APPENDIX Palivizumab Administration Recommendations from the American Academy of Pediatrics in 2006

- Infants and children less than 24 months of age with CLD of prematurity who have required medical therapy within the past 6 months

- Infants born at greater than 28 weeks and less than 32 weeks gestation with or without CLD who are less than 6 months of age at the start of the RSV season

- Infants born at 28 weeks of gestation or less with or without CLD and who are less than 12 months of age at the start of the RSV season

- Infants born between 32 and 35 weeks gestation without CLD who are less than 6 months of age and who have 2 or more of the following risk factors: school age siblings; day-care attendance; exposure to environmental air pollutants; congenital abnormalities of the airways; severe neuromuscular disease

- Infants and children 24 months of age and younger with hemodynamically significant cyanotic and acyanotic congenital heart disease; need for prophylaxis based on degree of physiologic cardiovascular compromise

- Infants less than 24 months of age with congenital heart disease that would benefit most from palivizumab include: infants receiving medication to control congestive heart failure; infants with moderate to severe pulmonary hypertension; infants with cyanotic heart disease

- For infants and children requiring cardiopulmonary bypass and receiving palivizumab, a postoperative dose should be considered; mean decrease in palivizumab serum concentration of $58 \%$ following procedures requiring cardiopulmonary bypass

a Excerpted from the Clinical Practice Guideline. Diagnosis and Management of Bronchiolitis (2006). ${ }^{13}$

$C L D=$ chronic lung disease $; R V=$ respiratory syncytial virus. 